\title{
pH-sensitive Pickering Emulsion Stabilized by Hydroxyapatite Nanoparticles: Stability and Controlled Release Study
}

\author{
Papa Mady Sy ${ }^{1}$, *, Louis Augustin Diaga Diouf ${ }^{1}$, Alphonse Rodrigue Djiboune ${ }^{1}$, \\ Sidy Mouhamed Dieng ${ }^{4}$, Mamadou Soumboundou ${ }^{3}$, Cecile Diop ${ }^{1}$, Toro Diop ${ }^{1}$, Gora Mbaye ${ }^{1}$, \\ Mamadou Mbodj', Mounibe Diarra, ${ }^{1}$, \\ ${ }^{1}$ Department of Pharmacy, Laboratory of Pharmaceutical Physics, Faculty of Medicine, Pharmacy and Odontology, Cheikh Anta Diop \\ University, Dakar, Senegal \\ ${ }^{2}$ Department of Medicine, Laboratory of Medical Biophysics and Nuclear Medicine, Faculty of Medicine, Pharmacy and Odontology, \\ Cheikh Anta Diop University, Dakar, Senegal \\ ${ }^{3}$ Department of Pharmacy, Service of Medical Biophysics, Health Training and Research Unit, Thies University, Thies, Senegal \\ ${ }^{4}$ Department of Pharmacy, Laboratory of biopharmacy, Health Training and Research Unit, Thies University, Thies, Senegal
}

\section{Email address:}

papamady.sy@ucad.edu.sn (P. M. Sy), mounibe.diarra@ucad.edu.sn (M. Diarra)

${ }^{*}$ Corresponding author

\section{To cite this article:}

Papa Mady Sy, Louis Augustin Diaga Diouf, Alphonse Rodrigue Djiboune, Sidy Mouhamed Dieng, Mamadou Soumboundou, Cecile Diop, Toro Diop, Gora Mbaye, Mamadou Mbodj, Mounibe Diarra. pH-sensitive Pickering Emulsion Stabilized by Hydroxyapatite Nanoparticles: Stability and Controlled Release Study. European Journal of Biophysics. Vol. 8, No. 2, 2020, pp. 52-59. doi: 10.11648/j.ejb.20200802.16

Received: October 21, 2020; Accepted: November 7, 2020; Published: November 19, 2020

\begin{abstract}
This study investigates the formulation of surfactant-free Pickering emulsions that release a drug at a specific $\mathrm{pH}$ to improve its oral bioavailability. The stabilizing nanoparticles composed of hydroxyapatite were obtained by a process of nanoprecipitation. Pickering oil-in-water emulsions stabilized with hydroxyapatite nanoparticles and encapsulating a hydrophobic drug model (ibuprofen) were formulated using a high-energy process with rotor-stator turbo mixer (IKA® T25 digital ultra-Turrax). The experimental approach explored the impact of all formulation parameters, dispersed phase and amount of hydroxyapatite nanoparticles on the physicochemical properties of Pickering emulsions. The system was characterized by a methylene blue test, $\mathrm{pH}$ and conductivity measurements, and droplet size determination. In addition, Pickering emulsions stabilized by hydroxyapatite nanoparticles have the advantage of being destabilized in acidic medium leading to the release of the active principle via the droplets. The acidic medium release study (pH equal to 1.2) showed ibuprofen release as a function of initial droplet loading and saturation concentration. In the simulated intestinal medium at $\mathrm{pH}$ equal to 6.8 , we found a better release of ibuprofen from emulsions that already had saturation in an acid medium. Thus, the interest of these Pickering emulsions lies on the fact that their non-toxicity and hydroxyapatite nanoparticles have advantage of being biocompatible because having the same mineral composition as bones and teeth. In addition, they allow destabilization of the emulsions and release of the drug. These emulsions not only protect patients from the side effects of acid-based drugs, but also contribute to increase the bioavailability of these acidic drugs.
\end{abstract}

Keywords: Emulsion, Pickering, Nanoparticles, Hydroxyapatite, Oral Bioavailability

\section{Introduction}

In many industrial sectors such as pharmacy [1], cosmetics [2], agri-food [3] and chemistry [4], emulsions are formulations used to obtain useful properties or suitable compositions. These emulsions are metastable dispersed systems consisting of least two immiscible liquids and an amphiphilic agent. One of the liquids is dispersed in the other 
in form of small spherical droplets whose size varies according to the conditions from 0.1 to a few tens of micrometers [5-7]. The system thus created does not correspond to a thermodynamically stable state, the most stable state would consist in the macroscopic separation of the two fluids. These metastable dispersed systems are conventionally stabilized by surfactant molecules. However, the demand for surfactants in the global economy is exponential growing $[8,9]$. Thus, it is necessary to reduce the use of surfactant in all these applications. On the one hand for the sake of environmental respect and on the other hand for the safety of consumers, surfactants can be toxic and harmful to the environment but also harmful to consumers $[10,11]$.

Ramsden and Pickering demonstrated at the beginning of the last century the feasibility of surfactant-free emulsions in presence of solid particles $[12,13]$. These emulsions are called "Pickering Emulsions". This concept of emulsions stabilized by solid particles is gaining renewed interest today because of the many advantages. It offers good stability, environmental protection, user safety, variety of particles, etc. In addition, one of the main advantages of Pickering emulsions is that they are more stable than other types of emulsions [12, 14, 15]. The adsorption of solid particles at the oil-water interface is almost irreversible and strong (unlike surfactants which are in thermodynamic equilibrium at the interface), leading to the formation of a dense film, creating a barrier around the droplets and thus making the droplets very resistant to coalescence. Recently, the applications of emulsions stabilized by solid particles are reconsidered in biopharmaceutics. This type of formulation are potential system of encapsulation of drugs, allowing the controlled and targeted release of the asset from the internal phase [5, $6,16,18]$.

An interesting approach would therefore be the formulation of a surfactant-free Pickering emulsion encapsulating drugs and then using a stimulus such as $\mathrm{pH}$ to establish controlled release systems. The formulation of $\mathrm{pH}$-dependent Pickering emulsions for controlled release of active substances to improve their oral bioavailability is precisely the general objective of this work. Thus, the interest of these Pickering emulsions lies on the fact that their non-toxicity and hydroxyapatite nanoparticles have advantage of being biocompatible because having the same mineral composition as bones and teeth. In addition, they also allow destabilization of the emulsions and release of the drug. These emulsions not only protect patients from the side effects of acid-based drugs, but also contribute to increase the bioavailability of these acidic drugs. In fact, hydroxyapatite nanoparticles once in the stomach can increase the $\mathrm{pH}$ and promote the release of active ingredients such as ibuprofen whose solubility is strongly influenced by $\mathrm{pH}$.

\section{Materials and Methods}

\subsection{Materials}

Potassium chloride, Methylene blue, sodium hydroxide, hydrochloric acid, potassium phosphate monobasic and potassium phosphate dibasic were purchased from SigmaAldrich. Calcium hydroxide $\left[\mathrm{Ca}(\mathrm{OH})_{2}\right] \cdot$ was purchased from Appli-chem GmbH. The oily phase used throughout the study is a peanut oil Niani ${ }^{\circledR}$ from the market (mainly composed of mono-unsaturated, polyunsaturated fatty acid and saturated fatty acid). The aqueous phase used is distilled water. Ibuprofen was purchased from FAGRON S.A (Saint-Denis, France). Acrodisc Syringe Filters with Nylon Membrane and dialysis membrane tubing (Spectra/Por molecular porous membrane tubing MWCO $12-14 \mathrm{kDa}$ ) were purchased respectively from PALL life sciences and Spectrum laboratories (USA). All the chemicals were analytical grade and used as received.

\subsection{Methods}

\subsubsection{Synthesis of Hydroxyapatite Nanoparticles}

Hydroxyapatite was produced by direct precipitation by adding with stirring a solution of phosphoric acid $0.3 \mathrm{M}$ $\left(\mathrm{H}_{3} \mathrm{PO}_{4}\right)$ at a rate of $25 \mathrm{ml}$ per minute to a freshly prepared suspension of calcium hydroxide $\left[\mathrm{Ca}(\mathrm{OH})_{2}\right] \quad 0.5 \mathrm{M}$. This precipitation reaction takes place between $80-95^{\circ} \mathrm{C}$. The powder precipitated was filtered and dried in an oven at $80^{\circ} \mathrm{C}$ for 24 hours. This powder has a specific surface of $40 \mathrm{~m}^{2} / \mathrm{g}$ and is formed of nanoparticles around 10 to $20 \mathrm{~nm}$ and flocs of nanoparticles around $1 \mu \mathrm{m}$ [19].

\subsubsection{Formulation of Pickering Emulsions}

During the formulation, the type of emulsions formed is one of the most important properties and characteristics. The Bancroft rule, which states that the type of emulsion depends on the medium in which the particles are introduced initially, served as a model for the preparation of the formulations.

(i) Preparation of the Dispersed Oil Phase

The amount of ibuprofen to be incorporated is dissolved in the peanut oil. The mixture is homogenized with a magnetic stirrer at $1000 \mathrm{rpm}$ for one minute.

(ii) Preparation of the Dispersing Aqueous Phase

In a distilled water, the hydroxyapatite nanoparticles were progressively added by stirring at $1680 \mathrm{rpm}$.

(iii) Emulsification

In the suspension previously prepared, the oily phase is gradually added followed by the fragmentation of the drops of oil with the mixer. Subsequently, the final mixture is homogenized vigorously for one minute at $5000 \mathrm{rpm}$. The total time of preparation of the emulsion is five minutes. The preparation of all the emulsions of this work was carried out under the same operating conditions (stirring speed, stirring time, type of stirrer, temperature). Thus, $\mathrm{O} / \mathrm{W}$ emulsions containing ibuprofen in the internal phase was realised. We prepared the Pickering emulsions in the following proportions (Table 1): 
Table 1. Proportions of formulations.

\begin{tabular}{|c|c|c|c|c|c|c|c|c|c|c|c|c|c|c|c|}
\hline Tubes & $\begin{array}{l}\text { TA } \\
\left(\mathbf{T A}^{\prime}\right) \\
\end{array}$ & $\begin{array}{l}\text { TB } \\
\text { (TB') }\end{array}$ & $\begin{array}{l}\text { TC } \\
\text { (TC') }\end{array}$ & $\begin{array}{l}\text { TD } \\
\text { (TD') }\end{array}$ & $\begin{array}{l}\text { TE } \\
\text { (TE') }\end{array}$ & $\begin{array}{l}\text { TF } \\
\text { (TF') }\end{array}$ & $\begin{array}{l}\begin{array}{l}\text { TG } \\
\text { (TG') }\end{array} \\
\end{array}$ & $\begin{array}{l}\text { T1 } \\
\text { (T1') }\end{array}$ & $\begin{array}{l}\text { T2 } \\
(\mathbf{T 2})\end{array}$ & $\begin{array}{l}\text { T3 } \\
\text { (T3') }\end{array}$ & $\begin{array}{l}\text { T4 } \\
\text { (T4') }\end{array}$ & $\begin{array}{l}\text { T5 } \\
\text { (T5') }\end{array}$ & $\begin{array}{l}\text { T6 } \\
\text { (T6') }\end{array}$ & $\begin{array}{l}\text { T7 } \\
\text { (T7') }\end{array}$ & $\begin{array}{l}\text { T8 } \\
\left(\mathbf{T 8}^{\prime}\right)\end{array}$ \\
\hline Oil (ml) & 15 & 15 & 15 & 15 & 15 & 15 & 15 & 8 & 9 & 10 & 11 & 12 & 13 & 14 & 15 \\
\hline Water (ml) & 15 & 15 & 15 & 15 & 15 & 15 & 15 & 22 & 21 & 20 & 19 & 18 & 17 & 16 & 15 \\
\hline Hydroxyapatite (g) & 0,2 & 0,4 & 0,6 & 0,8 & 1 & 1,50 & 2,00 & 1 & 1 & 1 & 1 & 1 & 1 & 1 & 1 \\
\hline
\end{tabular}

The notation prime is used when the emulsions contains additionally $\mathrm{NaCl}$ at a concentration of $5 \mathrm{mg} / \mathrm{mL}$ (TA', TB', TC', TD', TE', TF', TG', T1', T2', T3', T4', T5', T6', T7' and $\mathrm{T} 8$ ').

After preparation, each formulation was distributed into two tubes and stored at room temperature, protected from light for 28 days. The first tube is reserved for macroscopic examination and the second for the study of physicochemical characteristics. We first looked for the quantity of nanoparticles to be used with the tubes TA, TB, TC, TE, TF, TG, T'A, T'B, T'C, T D, T'E, T'F, T'G, which guided us to choose $1 \mathrm{~g}$ of hydroxyapatite in the tubes where we varied the volume fraction of dispersed phase (T1, T2, T3, T4, T5, T6, T7, T8, T1', T2', T3', T4', T5', T6', T7' and $\mathrm{T} 8^{\prime}$ ).

The incorporation of ibuprofen in the dispersed oily phase was carried out using the proportions of the tubes having a better stability after 28 days of follow-up (T3, T4, T5, T6, T3', T4', T5' and T6'). The formulation method remains the same except that here ibuprofen is dissolved in the oily phase before emulsification. The solubilization of ibuprofen in the oil was carried out using a magnetic stirrer (Table 2).

\subsubsection{Pickering Emulsion Stability Study}

\section{(i) Bottle Test}

The emulsions are conserved in the absence of light and an ambient temperature in $50 \mathrm{ml}$ conical bottle. This visual inspection makes it possible to demonstrate certain phenomena of instability such as sedimentation, flocculation, and coalescence.

(ii) Direction of the Emulsions

We used the dye test based on the determination of the solubility of the methylene blue in the emulsion obtained. Two milliliters of the emulsion were placed on a blade and mixed with a few milligrams of methylene blue. After assembly with a slide, observation will be done under optical microscope Axio Zeiss imager A1 coupled to a computer containing the Axio Vision release software Version 4.5 (Zeiss optical microscope).

(iii) Droplet Size Measurements

The technique used is based on the estimation of the mean diameter of the droplets by individual counting. The light chamber microscope BBT KRAUSS was used for measurements.

(iv) $\mathrm{pH}$ of the Emulsions

The measuring cell is introduced into a $50 \mathrm{ml}$ conical bottle containing the emulsion. Be sure to place the electrode at the emulsified phase for the sediment tubes. The reading time is set to three minutes after insertion of the electrode.

\subsubsection{Encapsulation Efficiency (E.E)}

The concentration of ibuprofen in each sample is determined following the measurement of the absorbance of ibuprofen in water at $222 \mathrm{~nm}$ by UV-visible spectrophotometry (Evolution $300 \mathrm{UV}$-visible). The equation of the following line (Eq.1) made it possible to determine the concentrations of ibuprofen in the external phase:

$$
\text { Abs. }=115,4 \mathrm{C}+0,1675 \text { with } \mathrm{R}^{2}=0,9938
$$

The encapsulation efficiency was calculated according to the following relation:

$$
\% E . E=\frac{[\text { ibuprofen total }]-[\text { ibuprofen externe }]}{[\text { ibuprofen Total }]} \times 100
$$

\subsubsection{In vitro Release Study Using $\mathrm{pH}$}

In vitro dissolution profiles of Pickering emulsions encapsulating ibuprofen were obtained using a dialysis membrane (12,000-14,000 Da). The dialysis tube containing $5 \mathrm{ml}$ of ibuprofen-loaded Pickering emulsions and $5 \mathrm{ml}$ of the dissolution medium was introduced into the in vitro release medium containing $250 \mathrm{ml}$ of the dissolving dissolution medium at $100 \mathrm{rpm}$. This dissolution medium consists of an acidic buffer solution simulating the gastric fluid at $\mathrm{pH}=1.2$ and a phosphate buffer solution simulating the intestinal fluid at $\mathrm{pH}=6.8$. Whole assemblies were maintained at a temperature of $37 \pm 1^{\circ} \mathrm{C}$. At 15 minutes intervals, $5 \mathrm{ml}$ samples of the dissolution medium were removed and analysed by UV-visible spectrophotometry. Sink conditions were maintained by replacing $5 \mathrm{~mL}$ of the release medium with $5 \mathrm{~mL}$ of fresh media at each sampling point. The percentage released in ibuprofen was obtained from the amount of ibuprofen initially present in the emulsions, compared to that measured in the release medium. For each point, three determinations were made.

\section{Results}

\subsection{Stability of Pickering Emulsions}

The study of the physicochemical and analytical parameters of the various emulsions formulated and stored in the absence of light at room temperature for 28 days, allowed us to follow the evolution of the formulations as a function of time.

\subsubsection{Bottle Test and Emulsion Direction}

The emulsions obtained are white and homogeneous. The emulsions are all stable, better stability was observed with the emulsions containing $1 \mathrm{~g}$ of HAP (Figure 1). 
The dye test carried out weekly during the 28 days of monitoring showed emulsions with oily droplets of heterogeneous sizes with a dispersant phase coloured blue by a hydrophilic dye (Methylene blue) (Figure 2).
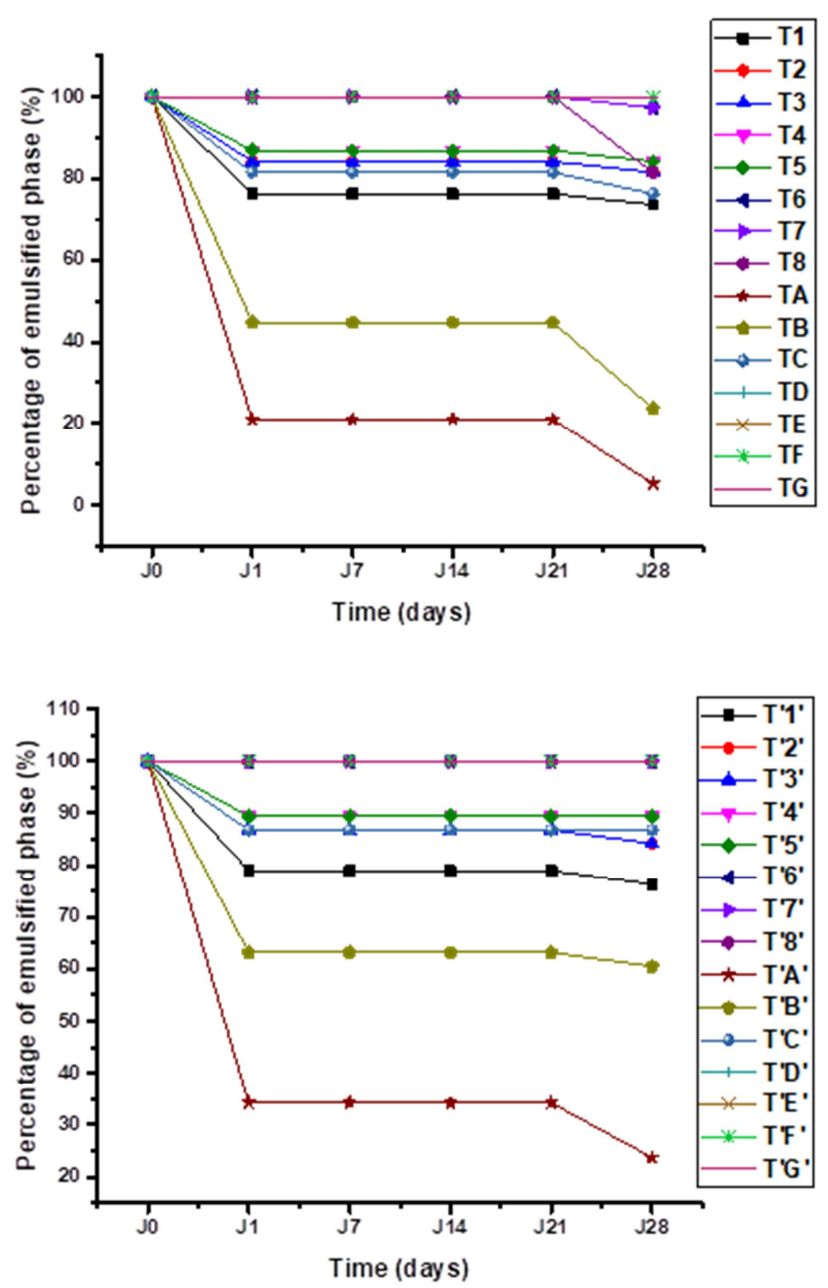

Figure 1. Percentage of emulsifying phase during the storage.

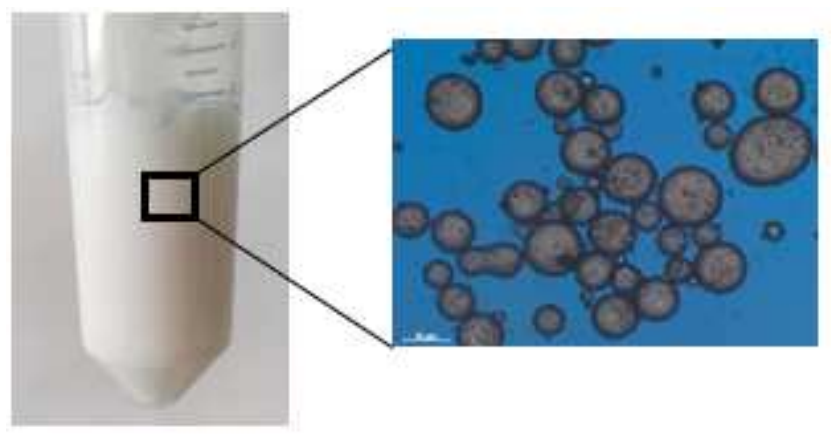

Figure 2. Aspect of tube T6 (1g of HAP) after 28 days of storage coloured by a hydrophilic dye (Methylene blue).

\subsubsection{Size of Droplets}

The size of the droplets varied according to the amount of HAP used (Figure 3). We found that the size of droplets decreased when the amount of hydroxyapatite increased.

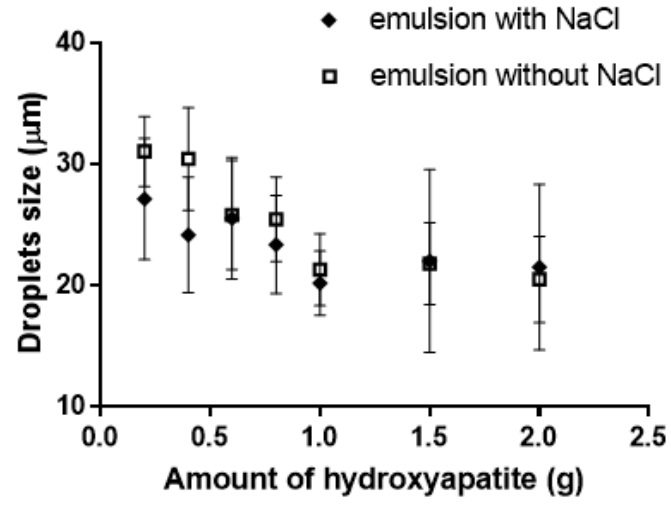

Figure 3. Evolution of the size of the droplets as a function of the amount of HAP.

\subsection{3. pH of the Emulsions}

Figure 4 shows the effect of the variation of the ratio $\mathrm{HAP} /$ dispersed phase on the $\mathrm{pH}$ values. We can find that the increase in the HAP / dispersed phase ratio did not change the $\mathrm{pH}$ values.

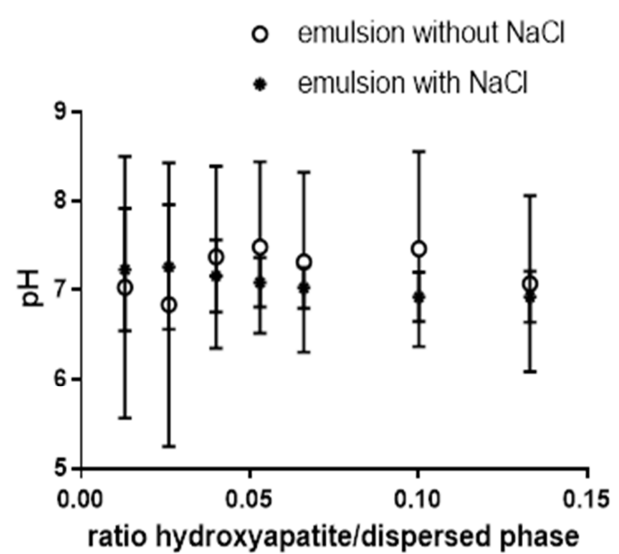

Figure 4. Evolution of the pH of emulsions depending on the ratio hydroxyapatite/dispersed phase.

\subsection{Encapsulation Efficiency of Ibuprofen}

The evaluation of the encapsulation efficiency (E.E) showed good encapsulation rates. Better rates were obtained in the most stable emulsions without $\mathrm{NaCl}$ (Figure 5).

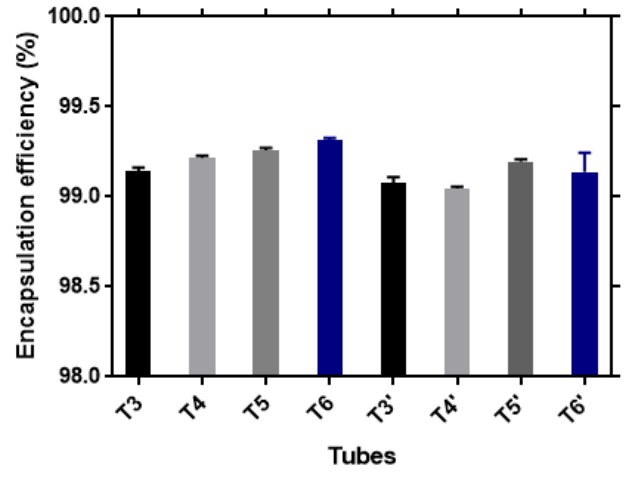

Figure 5. Encapsulation efficiency of emulsions. 


\subsection{In Vitro Release Study Using $p H$}

The choice of HAP nanoparticles was done because of their no toxicity and their capacity to be solubilized in the gastric medium ( $\mathrm{pH}$ equal to 1.2). The idea is to induce a specific destabilization of the droplets on function of $\mathrm{pH}$, leading to the emulsion destabilization and to the release of encapsulated ibuprofen. As can be seen from the Figure 6, the percentages of ibuprofen released were strongly dependent on the saturation concentration of the medium.

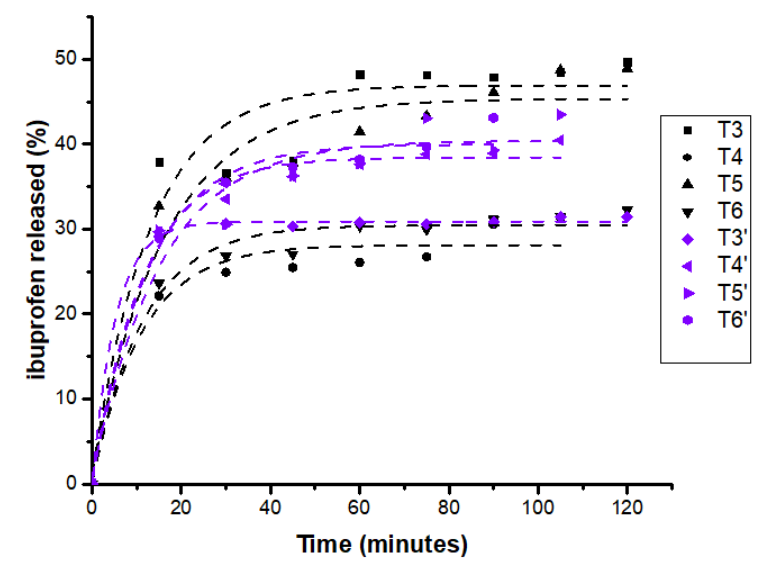

Figure 6. Ibuprofen dissolution profiles in an acid medium $(p H=1.2)$ as a function of time, modelled according to first order kinetics.

The modelling parameters are available in the supplementary information (Table 3 ).

The release of ibuprofen was also studied in phosphate buffer at $\mathrm{pH}=6.8$ simulating the intestinal fluid. This study was carried out after 120 minutes of residence in an acid medium corresponding to the residence time of the emulsion in the stomach (Figure 7). There was a significant release of ibuprofen from the emulsion that already had saturation in an acid medium. The greater release in this medium is strongly favored by a prior destabilization of the emulsions in an acid medium.

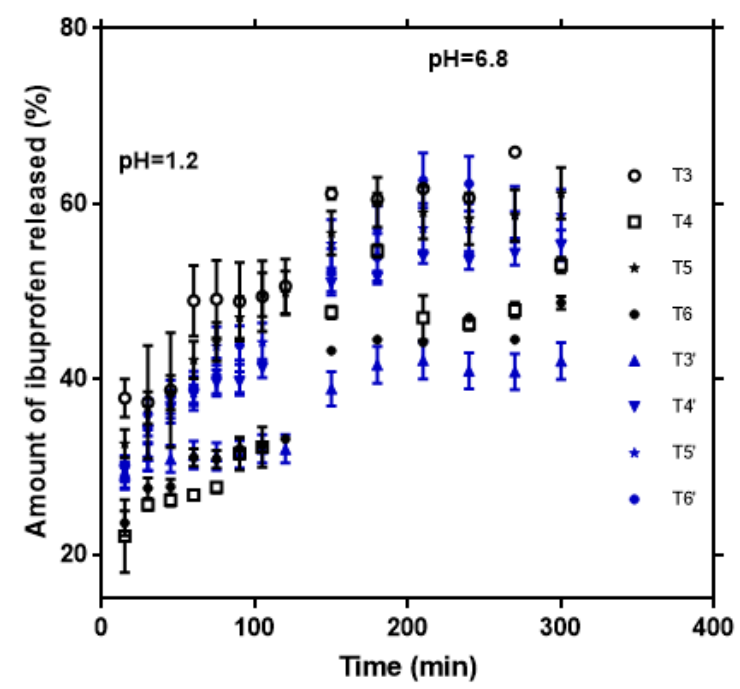

Figure 7. Dissolution profiles of ibuprofen in acid buffer at $\mathrm{pH}=1.2$ followed by a study in phosphate buffer at $p H=6.8$ for Pickering emulsion.

\section{Discussion}

The macroscopic examination showed homogeneous and stable emulsions for the majority with percentages of emulsified phases between 86.4 and $100 \%$ for the tubes TD, TE, TF, TG, T5, T6, T7, T8; emulsions with a creaming phenomenon with percentages between 76.32 and $86.84 \%$ for tubes T1, T2, T3, T4, TC and emulsions with a percentage of less than 50\% which decreases over time with the TA and TB tubes. A slight oily supernatant was observed on D28 in most of the tubes, due to a phenomenon of coalescence. The small percentage of emulsified phases observed in the TA and TB tubes can be explained by the small amount of particles used for their formulation. The same results are obtained for tubes with $\mathrm{NaCl}$ with higher percentages of emulsified phases. For example, at D1, the tube TA had a percentage of emulsified phase of $21.05 \%$ against $34.5 \%$ for the tube T'A. However, it should be borne in mind that the macroscopic observation alone does not predict the stability of the emulsion. Indeed, macroscopic observation does not allow to see oily droplets whose size is of the order of a micrometer.

The type of the emulsions was established by means of the methylene blue test and the measurement of the conductivity. The methylene blue test carried out under an optical microscope showed heterogeneous droplets dispersed in an external phase colored blue, thus indicating the $\mathrm{O} / \mathrm{W}$ nature of the emulsions. For all the formulations, we have obtained values near to water's conductivities values. Indeed, the value of the conductivity of an emulsion depends on its external phase [20] and the presence of electrolytes in this phase. The results thus obtained throughout the storage period show that the emulsions have not undergone any phase inversion phenomenon. In addition, the conductivity values of the external phase of the emulsion are lower than the normal value of the conductivity of water with or without $\mathrm{NaCl}$. Indeed, the oil being apolar, the oily droplets having a heterogeneous distribution in the dispersing phase will reduce the conductive role of the electrolytes of water and $\mathrm{NaCl}$. Similar results have been observed by ROJAS [21]. However, with regard to our study, the increase in the volume fraction of dispersed phase as well as the hydroxyapatite / fixed dispersed phase ratio did not influence the values of the conductivity. Furthermore, the conductivity values of emulsions containing $\mathrm{NaCl}$ ( $\mathrm{T}^{\prime}$ ) are much higher than those of emulsions without $\mathrm{NaCl}(\mathrm{T})$.

For the size of the droplets, it plays an important role in the stability of the emulsions. It is one of the variables that most influences the rate of sedimentation described by Stokes' law $[22,23]$. Thus, we studied the impact of the increase in the amount of particles on the evolution of the droplet size compared to a fixed volume of dispersed phase. We have seen a decrease in droplet size compared to an increase in the amount of particles. However, the relation which links the diameter of the droplets (D), the dispersed phase and the amount of particles is the following $[6,7,24]$ : 


$$
D=\frac{6}{\rho_{\text {oil }} \cdot A} \frac{m_{\text {oil }}}{m_{\text {particles }}}
$$

$\rho_{\text {oil }}=$ density of oil; $A=$ interfacial area covered by particles; $m_{o i}=$ masse of oil; $m_{\text {particles }}=$ masse of particles.

From this relationship we can say that the decrease in droplet size is due to an increase in the interfacial area covered by the particles combined with an increase in the amount of particles. We have also found that emulsions containing ibuprofen have much finer droplets than those without drug. This droplet size reduction could probably be explained by the hydrophobic properties of ibuprofen which would delay coalescence phenomena.

For the study of $\mathrm{pH}$, the results showed a basic character for all of emulsions without $\mathrm{NaCl}$ and $\mathrm{pH}$ values which tend towards neutrality for the emulsions containing $\mathrm{NaCl}$ when the volume fraction of the dispersed phase varied. We also noticed that the increase in the amount of hydroxyapatite compared to a fixed volume of dispersed phase did not change the $\mathrm{pH}$ values. Yang and al. had worked with LDH particles and had found that adjusting the $\mathrm{pH}$ to high values allowed good stabilization of the emulsion by promoting better adsorption of particles at the interfaces [25]. However, we noted a decrease in $\mathrm{pH}$ values for emulsions containing ibuprofen. This decrease in $\mathrm{pH}$ is no doubt due to the acidic nature of ibuprofen which has a very low solubility in water [26, 27].

About the study of encapsulation efficiency, the first remark is that we had obtained good encapsulation rates (Figure 5). In addition, these encapsulation rates were higher in tubes without $\mathrm{NaCl}$. The stability of the emulsions guarantees good protection of the droplets against coalescence. In addition, the low solubility of ibuprofen in water $(0.02 \mathrm{mg} / \mathrm{mL})$ means that it remains in the internal phase. Similar results were obtained by frélishowska and al. [6] with caffeine. One of the objectives of our work was also to study the release of ibuprofen in a simulated gastric fluid. The acidity of this medium was used as an external stimulus for the destabilization of emulsions. The percentage released

Supplementary Figure in ibuprofen was obtained from the amount of ibuprofen initially present into the emulsions, compared to that measured in the dissolution medium. For most tubes, the percentages released in ibuprofen were less than $50 \%$ of the initial load. Indeed, ibuprofen has a very low solubility in an acid medium $(0.06 \mathrm{mg} / \mathrm{mL})[26,27]$. As a result, the saturation concentration in the dissolution medium is quickly reached. Thus, several release profiles as a function of time were obtained. These profiles were modelled according to kinetics of the first order of equation $P_{t}=P_{0}+A$. exp ${ }^{K t}$ with determination coefficients close to 1 [19, 28, 29].

In the simulated intestinal medium at $\mathrm{pH}$ equal to 6.8 , we found a better release of ibuprofen from emulsions which already showed saturation in an acid medium. Thus, the interest of these Pickering emulsions is that they are able to increase the bioavailability ibuprofen. In fact, hydroxyapatite nanoparticles once in the stomach can increase the $\mathrm{pH}$ and promote the release of active ingredients such as ibuprofen whose solubility is strongly influenced by the $\mathrm{pH}[26,27,30]$.

\section{Conclusion}

In this study, we synthesized hydroxyapatite nanoparticles by a precipitation reaction. These nanoparticles were used to stabilize Pickering emulsions oil-in-water type. A model of lipophilic drug, ibuprofen, was incorporated into the oily phase. The interest of these Pickering emulsions lies in the absence of surfactants. In addition, hydroxyapatite has the advantage having the same composition as bones and teeth and non-toxic. It makes it possible to have stable Pickering emulsions and their destabilization in an acid medium and the release of the active ingredient orally. These emulsions can protect patients from the side effects of acid medicines through the basic properties of hydroxyapatite but also contribute to the increase of the bioavailability of these drugs. In fact, hydroxyapatite once in the stomach can increase the $\mathrm{pH}$ and promote the release of active ingredients such as ibuprofen whose solubility is strongly influenced by $\mathrm{pH}$.

\section{Size Distribution by Intensity}

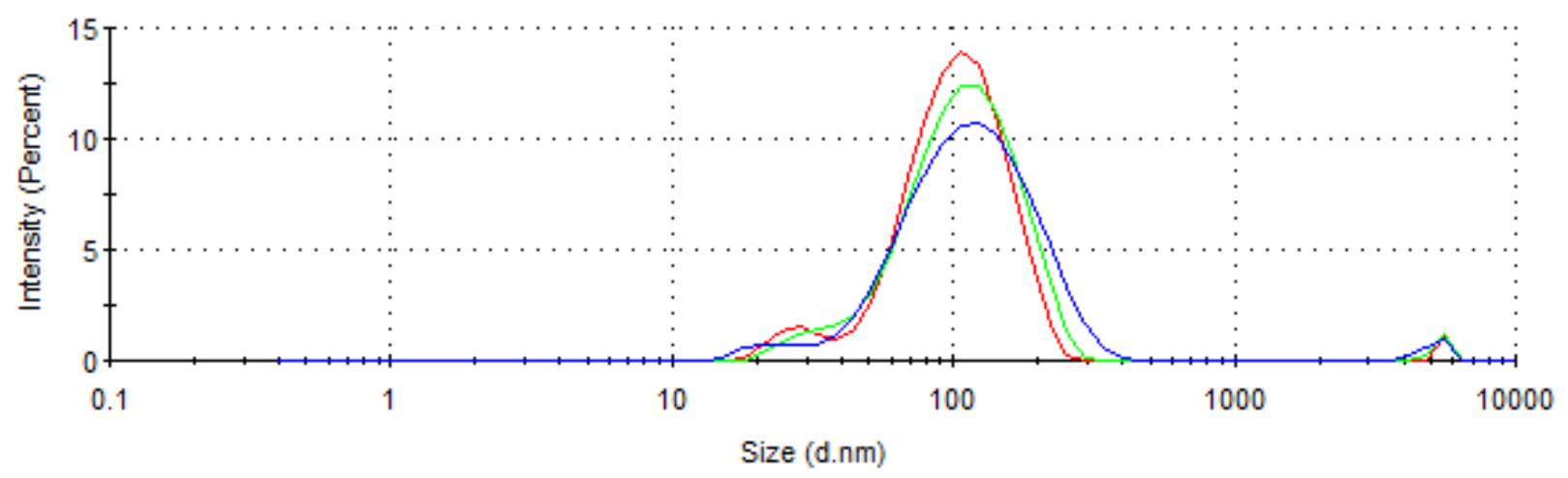

Figure 8. DLS characterization of hydroxyapatite nanoparticles. 
Table 2. Proportions of formulations containing ibuprofen.

\begin{tabular}{|c|c|c|c|c|c|c|c|c|}
\hline Tubes & T3 & T4 & T5 & T6 & T'3 & $T^{\prime} 4$ & T'5 & $T^{\prime} 6$ \\
\hline Water (ml) & 20 & 19 & 18 & 17 & 20 & 19 & 18 & 17 \\
\hline Oil (mL) & 10 & 11 & 12 & 13 & 10 & 11 & 12 & 13 \\
\hline Concentrations of ibuprofène $(\mathrm{mg} / \mathrm{mL})$ & 1,80 & 1,98 & 2,16 & 2,34 & 1,80 & 1,98 & 2,16 & 2,34 \\
\hline Hydroxyapatite (g) & 1 & 1 & 1 & 1 & 1 & 1 & 1 & 1 \\
\hline
\end{tabular}

Table 3. Parameters for modeling release profiles according to first order kinetics.

\begin{tabular}{|c|c|c|c|c|c|c|c|}
\hline & \multicolumn{2}{|l|}{$\mathbf{P}_{0}$} & \multicolumn{2}{|l|}{$\mathbf{A}$} & \multicolumn{2}{|l|}{$\mathbf{K}$} & \multirow{2}{*}{$R^{2}$} \\
\hline & Valeur & Ecartype & Valeur & Ecartype & Valeur & Ecartype & \\
\hline $\mathrm{T} 3$ & 46,91222 & 2,08342 & $-45,96256$ & 5,07064 & $-0,07698$ & 0,02181 & 0,90944 \\
\hline $\mathrm{T} 4$ & 28,12538 & 1,14099 & $-27,9336$ & 2,66308 & $-0,08987$ & 0,02369 & 0,93915 \\
\hline T5 & 45,35894 & 1,84673 & $-44,17049$ & 4,12373 & $-0,06147$ & 0,01399 & 0,93393 \\
\hline T6 & 30,47929 & 0,71208 & $-30,26307$ & 1,79195 & $-0,08677$ & 0,01383 & 0,97256 \\
\hline $\mathrm{T}^{\prime} 3$ & 30,89566 & 0,16144 & $-30,89497$ & 0,44938 & $-0,19007$ & 0,01684 & 0,99832 \\
\hline T'5 & 40,4282 & 1,88669 & $-39,57296$ & 3,91403 & $-0,06533$ & 0,01624 & 0,93472 \\
\hline T'6 & 39,9771 & 1,11865 & $-29,50574$ & 3,11236 & $-0,062$ & 0,015 & 0,98102 \\
\hline
\end{tabular}

\section{References}

[1] T. Hamouda, A. Myc, B. Donovan, A. Y. Shih, J. D. Reuter, et J. R. Baker, "A novel surfactant nanoemulsion with a unique non-irritant topical antimicrobial activity against bacteria, enveloped viruses and fungi”, Microbiological Research, vol. 156, n 1, p. 1-7, 2001, doi: 10.1078/0944-5013-00069.

[2] O. Sonneville-Aubrun, M. N. Yukuyama, et A. Pizzino, "Chapter 14 - Application of Nanoemulsions in Cosmetics", in Nanoemulsions, S. M. Jafari et D. J. McClements, Éd. Academic Press, 2018, p. 435-475.

[3] E. Dickinson, "Hydrocolloids as emulsifiers and emulsion stabilizers", Food Hydrocolloids, vol. 23, n 6, p. 1473-1482, august 2009, doi: 10.1016/j.foodhyd.2008.08.005.

[4] L. Ricaurte, M. Hernández-Carrión, M. Moyano-Molano, A. Clavijo-Romero, et M. X. Quintanilla-Carvajal, "Physical, thermal and thermodynamical study of high oleic palm oil nanoemulsions", Food Chemistry, vol. 256, p. 62-70, août 2018, doi: 10.1016/j.foodchem.2018.02.102.

[5] P. M. Sy et al., "Water/Oil Pickering Emulsion Stabilized by Magnesium Oxide Particles: A Potential System with Two Active Substances (Paracetamol and Griseofulvin)", Open Journal of Biophysics, vol. 08, p. 68, mars 2018, doi: 10.4236/ojbiphy.2018.82006.

[6] J. Frelichowska, M.-A. Bolzinger, J.-P. Valour, H. Mouaziz, J. Pelletier, et Y. Chevalier, "Pickering w/o emulsions: Drug release and topical delivery", International Journal of Pharmaceutics, vol. 368, $\mathrm{n}^{\circ}$ 1, p. 7-15, feb. 2009, doi: 10.1016/j.ijpharm.2008.09.057.

[7] J. Frelichowska, M.-A. Bolzinger, et Y. Chevalier, "Effects of solid particle content on properties of $\mathrm{o} / \mathrm{w}$ Pickering emulsions", Journal of Colloid and Interface Science, vol. $351, \mathrm{n}^{\circ}$ 2, p. 348-356, nov. 2010, doi: 10.1016/j.jcis.2010.08.019.

[8] P. T. Benvegnu et al., "Synthèses et propriétés de mélanges de nouvelles molécules polyfonctionnelles", p. 248.
[9] L. Ridel, "Pickering emulsions: theoretical approach and applications: physico-chemical analysis of interfacial phenomena: obtaining nanometric Pickering emulsions spontaneously and expanded Pickering emulsions", p. 299.

[10] Y. Cui, M. Threlfall, et J. S. van Duijneveldt, "Optimizing organoclay stabilized Pickering emulsions", Journal of Colloid and Interface Science, vol. 356, ${ }^{\circ} 2$, p. 665-671, avr. 2011, doi: 10.1016/j.jcis.2011.01.046.

[11] M. Errezma, A. B. Mabrouk, A. Magnin, A. Dufresne, et S. Boufi, "Surfactant-free emulsion Pickering polymerization stabilized by aldehyde-functionalized cellulose nanocrystals", Carbohydrate Polymers, vol. 202, p. 621-630, déc. 2018, doi: 10.1016/j.carbpol.2018.09.018.

[12] S. U. Pickering, "CXCVI.-Emulsions", J. Chem. Soc., Trans., vol. 91, $\mathrm{n}^{\circ}$ 0, p. 2001-2021, janv. 1907, doi: $10.1039 /$ CT9079102001.

[13] W. Ramsden, "Separation of solids in the surface-layers of solutions and 'suspensions' (observations on surfacemembranes, bubbles, emulsions, and mechanical coagulation).-Preliminary account", Proc. R. Soc. Lond., vol. $72, \quad \mathrm{n}^{\circ}$ 477-486, p. 156-164, janv. 1904, doi: 10.1098/rspl.1903.0034.

[14] M. Tang et al., "Au nanoparticle/graphene oxide hybrids as stabilizers for Pickering emulsions and $\mathrm{Au}$ nanoparticle/graphene oxide@polystyrene microspheres", Carbon, vol. 71, p. 238-248, mai 2014, doi: 10.1016/j.carbon.2014.01.034.

[15] S. Melle, M. Lask, et G. G. Fuller, "Pickering Emulsions with Controllable Stability”, Langmuir, vol. 21, n 6, p. 2158-2162, mars 2005, doi: 10.1021/la047691n.

[16] A. Salerno, M.-A. Bolzinger, P. Rolland, Y. Chevalier, D. Josse, et S. Briançon, "Pickering emulsions for skin decontamination", Toxicology in Vitro, vol. 34, p. 45-54, août 2016, doi: 10.1016/j.tiv.2016.03.005.

[17] P. M. Sy et al., "Pickering nano-emulsion as a nanocarrier for pH-triggered drug release", International Journal of Pharmaceutics, vol. 549, n ${ }^{\circ} 1-2$, p. 299-305, oct. 2018, doi: 10.1016/j.ijpharm.2018.07.066. 
[18] P. M. Sy et al., "Tramadol Encapsulation in Aqueous Phase of Water/Oil Pickering Emulsion Stabilized by Magnesium Oxide Particles", International Journal of Biochemistry and Biophysics, vol. 6, $\mathrm{n}^{\circ}$ 2, p. 37-43, avr. 2018, doi: 10.13189/ijbb.2018.060202.

[19] M. Diarra, G. Pourroy, D. Muster, M. Zingraff, et C. Boymond, "Elaboration and evaluation of an intraoral controlled release delivering system", Biomaterials, vol. 19, $\mathrm{n}^{\circ} 16$, p. 1523-1527, august 1998, doi: 10.1016/S01429612(98)00070-2.

[20] L. S. Dorobantu, A. K. C. Yeung, J. M. Foght, et M. R. Gray, "Stabilization of Oil-Water Emulsions by Hydrophobic Bacteria", Appl. Environ. Microbiol., vol. 70, n 10, p. 63336336, oct. 2004, doi: 10.1128/AEM.70.10.6333-6336.2004.

[21] R. Rojas et M. Patricia, "Agitated tank emulsification: role of the operating protocol on catastrophic phase inversion", thesis, Toulouse, INPT, 2007.

[22] D. Langevin, S. Poteau, I. Hénaut, et J. F. Argillier, "Crude Oil Emulsion Properties and Their Application to Heavy Oil Transportation", Oil \& Gas Science and Technology, vol. 59, $\mathrm{n}^{\circ}$ 5, p. 511-521, sept. 2004, doi: 10.2516/ogst:2004036.

[23] M. Seiller et F. Puisieux, Les systèmes dispersés. I, I,. Paris: Technique et documentation Lavoisier, 1983.

[24] Y. Chevalier et M.-A. Bolzinger, "Emulsions stabilized with solid nanoparticles: Pickering emulsions", Colloids and Surfaces A: Physicochemical and Engineering Aspects, vol. 439, p. 23-34, déc. 2013, doi: 10.1016/j.colsurfa.2013.02.054.
[25] F. Yang, Q. Niu, Q. Lan, et D. Sun, "Effect of dispersion pH on the formation and stability of Pickering emulsions stabilized by layered double hydroxides particles", Journal of Colloid and Interface Science, vol. 306, n 2, p. 285-295, févr. 2007, doi: 10.1016/j.jcis.2006.10.062.

[26] D. B. Jack, Handbook of Clinical Pharmacokinetic Data. Springer, 1992.

[27] M. Yazdanian, K. Briggs, C. Jankovsky, et A. Hawi, "The "High Solubility" Definition of the Current FDA Guidance on Biopharmaceutical Classification System May Be Too Strict for Acidic Drugs", Pharm Res, vol. 21, n² 2, p. 293-299, févr. 2004, doi: 10.1023/B:PHAM.0000016242.48642.71.

[28] M. Miastkowska, E. Sikora, J. Ogonowski, M. Zielina, et A. Łudzik, "The kinetic study of isotretinoin release from nanoemulsion", Colloids and Surfaces A: Physicochemical and Engineering Aspects, vol. 510, p. 63-68, déc. 2016, doi: 10.1016/j.colsurfa.2016.07.060.

[29] T. Higuchi, "Mechanism of sustained-action medication. Theoretical analysis of rate of release of solid drugs dispersed in solid matrices", Journal of Pharmaceutical Sciences, vol. 52, $\mathrm{n}^{\circ} 12, \quad$ p. 1145-1149, déc. 1963, doi: $10.1002 /$ jps.2600521210.

[30] P. M. Sy et al., "Formulation, Stability and Physicochemical Properties of Pickering Emulsions: An overview, Applied Physics Research, vol. 11, № $1, \quad 2019$, doi: 10.5539/apr.v11n1p41. 